\title{
ILCEA
}

Revue de l'Institut des langues et cultures

d'Europe, Amérique, Afrique, Asie et Australie

$28 \mid 2017$

Passages, ancrage dans la littérature de voyage

\section{Analyse du discours de la liminalité : Butler, de l'autre côté du Saint-Gothard entre passage et ancrage}

Analysing the Discourse of Liminality: Butler, over the Saint Gothard between Crossing and Anchorage

\section{Samia Ounoughi}

\section{OpenEdition}

Journals

Édition électronique

URL : https://journals.openedition.org/ilcea/4099

DOI : 10.4000/ilcea.4099

ISSN : 2101-0609

Éditeur

UGA Éditions/Université Grenoble Alpes

Édition imprimée

ISBN : 978-2-84310-374-2

ISSN : 1639-6073

\section{Référence électronique}

Samia Ounoughi, «Analyse du discours de la liminalité : Butler, de l'autre côté du Saint-Gothard entre passage et ancrage », ILCEA [En ligne], 28 | 2017, mis en ligne le 06 mars 2017, consulté le 07 décembre 2022. URL : http://journals.openedition.org/ilcea/4099; DOI : https://doi.org/10.4000/ilcea. 4099

Ce document a été généré automatiquement le 29 septembre 2020

Tous droits réservés 


\title{
Analyse du discours de la liminalité : Butler, de l'autre côté du Saint- Gothard entre passage et ancrage
}

\author{
Analysing the Discourse of Liminality: Butler, over the Saint Gothard between \\ Crossing and Anchorage
}

Samia Ounoughi

« Le monde est impossible à reprographier. Ce qu'un récit de voyage, ou plus généralement un texte référentiel, raconte, c'est aussi l'histoire, toujours rejouée, d'un permanent réajustement du

langage et du monde. » (Viviès, $2002: 34$ )

\section{Introduction}

Entre 1865 et 1902, Samuel Butler (1835-1902) passe presque tous ses étés dans le canton du Tessin et dans le Piémont italien. De ces voyages itérés, il a gardé des notes illustrées par des dessins et des partitions musicales qu'il a repris pour produire Alps and Sanctuaries of Piedmont and the Canton Ticino (1881) ${ }^{1}$. Cet ouvrage retrace, comme le récit d'un seul voyage, les voyages de Butler au-delà du Saint-Gothard. Sans prétention scientifique ni littéraire, Butler offre tout simplement sa vision du col du Saint-Gothard et du Val Léventine. L'intérêt majeur de cet ouvrage, tant dans le discours que dans les illustrations, est d'offrir des modalités nouvelles d'appréhension de cette zone peu fréquentée, contre un tourisme qui uniformise le vécu du voyageur: "Alps and Sanctuaries is thus more than a signpost in the history of anti-tourism; it challenges the intellectual foundations of the structural Continental tour by encouraging the traveller to see for himself rather than 'by the book'. (Zdanski, 2007 : 230). Entre le col du Saint-Gothard et le Val Léventine, Butler construit un territoire bien à lui au fil de la plume. Son texte, parfois déroutant, nous invite à découvrir comment il décrit et délimite une zone de passage dans laquelle il vient errer et qu'il s'approprie. Son écriture a une valeur 
performative de libération d'une vision imposée de la géographie locale pour lui substituer une expérience individuelle et, avec elle, un point de vue unique, notamment sur les notions de frontière et de territoire.

Ce travail s'inscrit dans l'un des axes de recherche du LABEX pluridisciplinaire ITEM (Innovation et Territoires de Montagne) qui explore notamment les notions de frontière et de limite dans son projet TeLiMéP: Territorialité, Liminalité et Métropolisation Périphériques. Dans ce groupe, «[1]es transformations de la montagne seront analysées à travers les dynamiques collectives des territoires, en lien avec les problématiques liées aux frontières et aux limites de tous ordres, aboutissant à une meilleure connaissance des territorialités en jeu et des reterritorialisations progressives ${ }^{2}$. " Aux côtés d'historiens et de géographes, ma recherche consiste à analyser, par les outils de la pragmatique, le discours des voyageurs britanniques dans les Alpes au XIX ${ }^{e}$ siècle, en particulier sur la traversée des cols, hauts lieux de liminalité-liminarité.

3 Le concept de liminalité ou de liminarité, énoncé par l'anthropologue Van Guennep en 1909 dans Rites de Passage désigne un entre-deux, un lieu et/ou un moment où une personne se trouve entre deux états, à un stade charnière où ses repères se brouillent alors qu'elle ne s'est pas encore reconstruite. C'est ce passage, où une personne n'est alors plus celle qui s'est séparée de son environnement et de son groupe social (séparation), ni encore celle qui, au terme d'une expérience de l'autre et de l'ailleurs viendra s'agréger à nouveau dans la société en tant qu'individu sinon totalement construit, au moins évolué (agrégation/ancrage). Parmi les phases de liminarité comme la traversée d'une rivière ou le passage d'une poterne de murailles, Van Guennep signale le passage des cols de montagne: «Parmi les rites de passage matériel il convient encore de citer ceux du passage des cols et qui comprennent le dépôt de divers objets [...], des offrandes, des invocations au génie du lieu, etc. : [...], Alpes (chapelles), etc. » (1981:29).

$4 \quad$ Le $\mathrm{XIX}^{\mathrm{e}}$ siècle britannique voit émerger la géographie comme discipline universitaire à part entière et à cette même période, le voyage en Europe se démocratise. Bien que le toit de la Grande-Bretagne, le Ben Nevis, culmine à moins de $1400 \mathrm{~m}$, les Britanniques sont les inventeurs de l'alpinisme et du terme qui désigne cette discipline. Quelle créativité linguistique et narrative leur a été nécessaire pour exprimer en anglais ce qui n'existait pas dans leur environnement direct? Enfin, le $\mathrm{xIx}^{\mathrm{e}}$ siècle est une période de développements infrastructurels majeurs qui changeront en profondeur la géographie des Alpes et la notion même de passage par la construction de voies carrossables, de chemins de fer et de tunnels pour aller de l'autre côté de la montagne. Ces questionnements se prolongent jusqu'à nos jours avec des projets comme celui de la ligne de chemin de fer à grande vitesse entre Lyon et Turin, la TAV.

Ainsi donc, les notions de frontières, de passage et bientôt d'ancrage se mettent en mouvement; elles se redéfinissent mutuellement, stimulant la créativité littéraire, comme chez Dickens (Ounoughi, 2016) et Collins, par exemple (Ounoughi, 2013a), ou la créativité artistique (comme chez Turner et Ruskin). Enfin, elles font évoluer la pensée géographique, donnant à concevoir le monde différemment à partir d'une expérience de l'ailleurs qui n'est pas seulement un déracinement, mais qui se prolonge dans un retour vers le point de départ, lui-même repensé et modifié autant que change le voyageur à mesure qu'il chemine au-delà des cols alpins les plus fréquentés. La problématique du retour a notamment été explorée par Jean Viviès (2016). Cette évolution de la façon dont nous pensons la géographie n'est pas à sonder uniquement chez les géographes euxmêmes, d'autant qu'à cette époque, leur discipline est encore naissante. On ne sait pas 
encore comment s'organise la géographie des montagnes (Debarbieux, 2008). Des sources documentaires éparses, dont les récits de voyageurs, intéressent aujourd'hui les géographes, notamment l'approche constructiviste qui met le sujet observateur au centre de leur réflexion.

L'analyse du discours a déjà révélé les enjeux majeurs du passage d'un col comme le Simplon ou le Grand-Saint-Bernard. Ces enjeux sont notamment d'ordre identitaire, pour celui qui en fait l'expérience (Ounoughi, 2013a, 2016). Ils procèdent aussi d'un remodelage de la géographie du col et avec lui toute une conception de la montagne par l'écrivain voyageur (Ounoughi, 2016). C'est pour approfondir cette dernière perspective que nous proposons ici l'exploration d'un texte peu connu, Alps and Sanctuaries of Piedmont and the Canton Ticino de Samuel Butler. À travers une analyse de la composition structurelle et stylistique de ce récit, nous tenterons de montrer comment l'expérience et la libre pensée de ce voyageur contribuent à une refonte des frontières et de la cartographie au-delà du col du Saint-Gothard.

7 Répondant à un besoin d'isolement, Butler déconstruit et reconstruit cette région du monde dans une itération de phases de séparation, de liminalité et d'agrégation, destinées à la perpétuelle reconstruction du voyageur et à la personnalisation de la géographie locale. Comment l'écriture de Butler entre le Saint-Gothard et le Piémont faitelle de ce val un espace-frontière?

\section{Redéfinir le col alpin : Butler, libre pensée et alpinisme}

\section{La remise en question d'une éducation et l'appel de la montagne}

Le sujet géographe importe autant que l'objet géographique comme le montre l'approche constructiviste du géographe Debarbieux :

En d'autres termes, le monde n'est pas fait d'objets géographiques que la géographie aurait pour mission de découvrir, sous l'apparence parfois trompeuse des phénomènes; il est fait de « choses » que le géographe compose en objets et en système d'objets dans le but d'en construire une connaissance. (2004:12)

S'il n'est pas question ici de faire une biographie détaillée de Samuel Butler, parcourons néanmoins quelques aspects de son existence qui éclairent sa démarche dans les Alpes et sa manière de les mettre en discours. L'expérience de terrain est fondamentale chez Butler. Dans les quartiers pauvres de Londres, alors qu'il se forme à devenir homme d'église, dans la lignée de son père et de son grand-père, Samuel Butler confronte sa perception du monde aux enseignements qui lui ont été prodigués. Les doutes qui le saisissent auront raison de sa vocation. Ainsi, l'observation de la réalité de terrain qui anime le libre-esprit de Butler se manifeste pour la première fois et marquera toute son existence ; elle catalysera sa pensée, ses voyages et son discours.

Butler quitte le foyer familial pour la Nouvelle-Zélande où il restera plus de quatre ans. Ses qualités sportives de coureur de fond et de grimpeur déjà reconnues à Cambridge serviront son âme d'explorateur, en particulier, dans la montagne. Son nom figure aujourd'hui sur les cartes géographiques de la Nouvelle-Zélande avec notamment, Butler Saddle, Mount Butler et Butler Range. On lui doit également la découverte du col de Whitcombe en 1861. Butler a une âme de pionnier; il explore les terres sans propriétaire et ses expéditions sont souvent des réponses à un appel de la montagne. Or, «La montagne est [donc] d'abord et avant tout un objet qui se différencie pour des raisons 
essentiellement topographiques de l'endroit d'où on la nomme. Elle est donc ailleurs; et elle est autre, autrement constituée, autrement organisée.» (Debarbieux, 2001:36). Le voyage en montagne permet littéralement à Butler de prendre de la hauteur et de revisiter sa pensée sociale, esthétique et spirituelle, la montagne étant un lieu particulièrement propice à la spiritualité (Debarbieux, 2001 : 36-38).

\section{Les cols de montagnes : entre passage et rupture} utopie satyrique Erewhon; Or, Over the Range (1872), qui lui vaut la réputation d'alpiniste libre-penseur dont l'histoire a retenu le nom. Ce texte dépeint bien la démarche de Butler qui, pour mener une critique sociale, imagine une civilisation aux règles absurdes, au-delà d'un col de montagne où lui seul a pu accéder. Il rappelle en ceci le voyage de Gulliver chez les Brobdingnagians ${ }^{3}$.

Les cols de montagne fascinent donc Butler car ils lui ouvrent la perspective d'un monde qu'il veut construire. Dans la vie, comme dans ses textes, ses capacités d'explorateur et d'alpiniste lui permettent de traverser ces passages réservés à quelques courageux des No Thoroughfare [s] comme les définit Dickens ${ }^{4}$. Frontière naturelle, le col de montagne est pour Butler à la fois un lieu de passage et un point de rupture avec son environnement quotidien de Londres. Dans Erewhon, le narrateur homodiégétique explique pendant quelques chapitres le périple au terme duquel il se retrouve seul à faire la traversée dangereuse d'un col avant de passer à la narration de la vie de l'autre côté du col, qui elle, est le cœur de son récit. En revanche, dans Alps and Santuaries, Butler s'intéresse surtout au monde qu'il explore dans le canton du Tessin et dans le Piémont italien. Il ne dédie que quelques lignes au col du Saint-Gothard dont la traversée apparaît d'emblée comme un passage désagréable qu'il a hâte de franchir pour parvenir au monde qu'il convoite :

True, by making use of the tunnel one will miss the St. Gothard scenery, but I would not, if I were the reader, lay this too much to heart. Mountain scenery, when one is staying right in the middle of it, or when one is on foot, is one thing, and mountain scenery as seen from the top of a diligence very likely smothered in dust is another. Besides I do not think he will like the St. Gothard scenery very much. (22)

13 Cette entrée en matière pose un contrat de lecture par lequel Butler guide le lecteur et impose sa réception du col de montagne. Butler préfère ici le tunnel au col dont il attend l'ouverture avec impatience. Or, le tunnel va modifier la géographie locale, raccourcir le voyage, éviter des paysages et faciliter la traversée. L'enthousiasme de Butler face à un changement d'une telle envergure vient renforcer l'idée que son expérience du voyage dans les Alpes ne tient pas au passage d'un col. En ceci, il se distingue d'une conception de la traversée du col comme une deuxième naissance, ce qui est le cas dans son Erewhon; Or, Over the Range et ailleurs chez Dickens dans No Thoroughfare (col du Simplon), Little Dorrit (col du Grand-Saint-Bernard) ou encore chez Ann Radcliffe dans The Romance of the Forest (Ounoughi, 2016).

\section{Passage du col et voyage à pied : du passage à l'ancrage}

Butler généralise l'absence d'intérêt qu'il voit dans le passage du Saint-Gothard à tous les cols alpins : «one pass will do as well as another » (25). Il ne valorise que les déplacements à pied comme seules véritables expériences du voyage en montagne. Cependant, malgré son désintérêt affirmé pour le col de montagne et en particulier pour le Saint-Gothard, 
Butler place celui-ci à l'ouverture et à la fin de son ouvrage, des points d'ancrage stratégiques de tout texte. Le passage du col de montagne délimite l'entrée en discours et le début de l'exploration. De la même manière, il signe la fin du texte et avec elle, la fin de son périple. Ceci s'explique car la traversée du Saint-Gothard se fait en diligence. Butler ne peut pas y faire son expérience propre et le col n'a donc que peu d'intérêt. Nul ne saurait en effet saisir «l'horizon immobile» (2003: 225) que décrit Daniel Roche sans aller à pied : « le voyageur commun choisit la voiture pour gagner du temps, le cheval qui joint l'utile à l'agréable, ou encore d'aller à pied parce qu'on est libre [...], qu'on accepte de s'égarer.» (2003: 225). La marche en montagne et l'acte d'écriture vont ainsi de pair, donnant une cohérence au mouvement du pied et de la plume, le discours sur la montagne et la marche se remodelant mutuellement en permanence. Butler présente donc le col comme un lieu de liminalité particulier. Ce n'est pas une traversée qui permet au voyageur de changer et de se redécouvrir, mais plutôt un point de rupture entre deux mondes, exprimé dans une quasi ellipse narrative dont la brièveté vient justement en souligner à la fois la nécessité et l'absence d'intérêt.

15 À son retour, le passage du col est aussi le dernier passage de son voyage qu'il prend la peine de narrer : « From Faiso we returned home. We looked at nothing between the top of the St. Gothard Pass and Boulogne, nor did we again begin to take any interest in life till we saw the science-ridden, culture-ridden, afternoon-tea-ridden cliffs of old England rise upon the horizon. " (303-4). De l'autre côté de la montagne, entre le Saint-Gothard et Londres, Butler inscrit un vide narratif, comme si ces deux points d'ancrage étaient séparés par un gouffre et que seul le col de montagne signalait la phase de séparation. Ce genre de cartographie narrative est typique du texte de Butler qui met ainsi en exergue sa proximité de cœur avec la montagne et raccourcit les distances géographiques par l'emploi d'une ellipse ou d'un résumé lapidaire.

\section{Une géo-graphie libérée : le refus des canons scientifiques et littéraires}

\section{La détermination de l'objet géographique par le plaisir}

Dans l'exploration de son Italie à petite échelle, Butler pérégrine de manière aléatoire. Il est donc difficile de le suivre, tant dans l'itinéraire que dans la composition de son discours. Où va-t-il et qu'est-ce qui motive son choix ? Là où les voyageurs de l'époque suivent un guide comme le Murray's pour visiter les hauts lieux de l'art italien et pour admirer les reliefs et les dépressions spectaculaires des Alpes, Butler suit une logique fondée uniquement sur la notion de plaisir. Il redéfinit la notion historique de voyage en dissociant travel et travail, le voyage comme une souffrance, arguant, au contraire :

I once heard a man say the only test was to ask one's self whether one would care to look at it if one was quite sure that one was alone; I have never been able to get beyond this test with the St. Gothard scenery, and applying it to the Devil's Bridge, I should say a stay of about thirty seconds would be enough for me. (25)

Plus loin, il ajoute :

Years ago, in travelling by the St. Gothard road, I had noticed the many little villages perched high up on the sides of the mountain, from one to two thousand feet above the river, and had wondered what sort of places they would be. I resolved, therefore, after a time to make a stay at Faido and go up to all of them. I carried out my intention, and there is not a village nor fraction of a village in the Val Leventina from Airolo to Biasca which I have not 
inspected. I never tire of them, and the only regret I feel concerning them is, that the greater number are inaccessible except on foot, so that I do not see how I shall be able to reach them if I live to be old. These are the places of which I do find myself continually thinking when I am away from them. (27-28).

Butler détermine son objet géographique selon un rapport de proportions qu'il établit entre ennui et plaisir. Dans son texte, ces deux notions recouvrent exactement celles de passage et d'ancrage. Il délimite donc un espace qui s'étend de la sortie du col du SaintGothard jusqu'au sud du Val Léventine à la frontière entre le canton suisse du Tessin et le Piémont italien.

Les voyages de Butler n'ont rien du Grand Tour. Il passe la plus grande partie de ses séjours entre Airolo et Biasca, deux villages du Tessin situés à $38 \mathrm{~km}$ de distance. Puis, il prolonge un peu ses excursions dans le Piémont. C'est dans ce court axe nord-sud très étroit que Butler vit une errance quotidienne dans des villages souvent absents des guides de voyages de l'époque, tels que le Murray's. C'est cet isolement qu'il recherche avant tout. $\mathrm{Du}$ voyage, rien n'est narré avant le col et au sud, sa narration s'arrête juste après la frontière italienne. Ainsi, sous sa plume, le Val devient un espace-frontière, une zone de liminalité qu'il explore et définit à sa guise.

Butler s'installe quelques jours dans un village à partir duquel il randonne et dessine. La présentation fragmentaire et fragmentée de ses excursions disperse ces villages pourtant très proches et donne dans le discours une amplitude exacerbée à ses errances, de sorte que la ligne devient un espace. Cet effet est intensifié par le découpage en 26 chapitres dont les titres signalent en majorité un ou plusieurs de ces petits villages qui atomisent d'autant les étapes des voyages. Enfin, Butler ne crée pas non plus d'intrigue qui donnerait une ligne directrice à son ouvrage, ce qui contribue également à cet effet de dispersion et de vaste étendue alors que l'on est à une petite échelle géographique.

21 Alps and Sanctuaries se distingue également par sa structure composite. Le texte est accompagné d'illustrations dessinées par l'auteur ainsi que de partitions musicales citées de l'œuvre de Händel et plus rarement, de son invention. Les dessins et les partitions signalent des points d'achoppement de l'intérêt de Butler, le plaisir étant le seul déterminant de son itinéraire et de son écriture :

As for knowing whether or not one likes a picture, which under the present aesthetic reign of terror is de rigueur, I once heard a man say the only test was to ask one's self whether one would care to look at it if one was quite sure that one was alone; I have never been able to get beyond this test with the St. Gothard scenery, and applying it to the Devil's Bridge, I should say a stay of about thirty seconds would be enough for me. (23)

\section{Le dessin et la musique : pour une nouvelle cartographie de l'espace et du discours}

L'attrait de Butler est avant tout esthétique, mais ne répond à aucun canon particulier, ni à aucun projet précis. Ainsi s'explique la contradiction entre le titre qui annonce une réflexion spirituelle et le texte dans lequel Butler évite les débats religieux et dessine les églises qui ont retenu son attention sans jamais débattre de la foi ni du christianisme, ni même de l'architecture. Les dessins composés au fil de ses excursions représentent de petites églises et des chapelles vues de loin et quelques dessins montrent des vues à l'intérieur des églises. Ce sont des dessins épurés, parfois schématiques, qui proposent pour la plupart la représentation d'une église dans le relief sur laquelle elle est posée. Butler y ajoute parfois une présence humaine, et lorsqu'il y a effectivement quelqu'un, les 
visages sont presque imperceptibles et les personnages affairés à leurs tâches quotidiennes ne sont jamais représentés en train de prier. A l'instar de son récit atomisé passant sans transition de l'entomologie à l'architecture ou à la botanique, les dessins représentent des détails qui se détachent de leur contexte et qui ont simplement retenu l'attention de Butler. Cette errance ponctuée de pauses inspirées par la curiosité ou l'attrait pour un détail sont les seuls éléments qui cartographient ses randonnées en montagne et les lieux qu'il visite.

Le texte de Alps and Sanctuaries est une synthèse de notes éparses que Butler a prises pendant ses séjours itérés tous les étés à partir du milieu des années 1860 jusqu'à la fin de sa vie. Il les a ensuite reprises et compilées pour obtenir cet ouvrage que Streatfield, auteur de l'introduction, nomme " $a$ holiday book». Jean Viviès rappelle d'ailleurs le caractère recomposé du récit de voyage, un travail qui remodèle nécessairement la structure du texte et la composition géographique qui en est l'objet (2015). L'appellation "récit de vacances» est importante car elle correspond parfaitement à la liberté de forme que Butler donne à son texte, un discours de l'errance qui se traduit dans une errance du discours. Au terme de cette succession de séjours dans le Val Léventine, le texte finit par relever moins de l'invention que de l'inventaire pour reprendre au sens littéral les termes d'Adrien Pascali (1984). Car enfin, et on le comprend après la lecture de quelques pages, Samuel Butler, pourtant petit fils de géographe, n'écrit pas pour rendre compte de la géographie physique du Val Léventine, mais pour s'approprier cette axefrontière et en faire son espace.

Le récit du vacancier-écrivain se distingue d'abord par un lissage total de la temporalité qui ne laisse aucune trace de l'itération de ses séjours, ni de repère chronologique : « For the convenience of avoiding explanations, I have treated the events of several summers as though they belonged to only one. This can be of no importance to the reader, but as the work is chronologically inexact, I had better perhaps say so." (11). Cette remarque métatextuelle annonce une manipulation de la temporalité que Butler définit comme une volonté de simplification. Cependant, l'effacement de l'itération des voyages et du temps qui passe occulte la notion d'évolution du voyageur et d'évolution de l'espace qu'il revisite année après année. Ce procédé va au-delà de la désinvolture; il modifie en profondeur la conception de cet espace qui n'existe plus dès lors que dans les notes du vacancierécrivain.

L'effacement de la temporalité ramène l'ensemble de ces voyages à une grande phase de liminalité. La non-narration de l'itération efface aussi les phases de séparation et les phases d'agrégation (le départ d'Angleterre et le retour). Finalement, le début et la fin de l'ouvrage signalent le passage du col du Saint-Gothard et le corps du texte la phase de liminarité. Il y a donc une inversion car la phase de liminalité qui est censée rester transitoire cesse d'être un lieu de passage et devient une zone d'ancrage. Butler nous propose une liminalité étendue qui correspond sans doute au concept de vacances qui ne signifie pas seulement le repos, mais la vacance, le lieu vide, cet espace frontière que Butler a à cœur de recréer. Ceci donne une vision renouvelée de la notion même de territoire.

La temporalité du récit et celle de ses errances sont rythmées par les musiques que Butler inclut dans ses notes et reporte dans son ouvrage. Butler insère une partition selon l'inspiration du moment, selon ce qui retient son attention et qui produit une analogie avec une musique qu'il connaît: «If there is a stream it will run with water limpid as air, and full of dimples as in 'White Kedron's Brook' in 'Joshua' » (260), puis il insère la partition dans 
son texte. Ces intermèdes musicaux se situent à des points d'achoppement dans l'errance de Butler. La musique y est avant tout suscitée par l'itinéraire du voyageur dont elle finit par rythmer le discours. Cette gestion de la temporalité du discours par la musique mériterait à elle seule une étude approfondie car elle propose une esthétisation du temps qui rompt avec les canons de la narration prosaïque et invite le lecteur à séquencer le voyage en mouvements musicaux inspirés au fil du voyage. Le lecteur est ainsi amené à abandonner tout un système de structuration de la pensée situé au fondement de ses repères temporels. Au fil de la lecture, il vit l'errance de Butler qui lui propose une vision renouvelée de cet espace-frontière à travers les modalités discursives qu'il a inventées.

Cette dispense de repères temporels se prolonge dans une quasi-disparition des repères spatiaux. Après être descendu du col du Saint-Gothard, Butler erre dans le Val Léventine dont il ne parcourt que $38 \mathrm{~km}$ sur 54 . L'ouvrage pourtant très illustré de Butler ne comporte aucune date ni aucune carte géographique. Les illustrations, quant à elles, fournissent une somme de détails qui jalonnent un espace dont le lecteur n'a jamais une vue d'ensemble. Aussi son texte apparait-il comme une construction aléatoire et donc difficile à appréhender, contrairement à ses dires. Ce discours de l'errance permet à Butler de se défaire d'emblée de toute rigueur scientifique et de tout canon prosodique. En ce sens, l'écriture de Alps and Santuaries aussi bien que l'expérience de Butler sur le terrain, rappellent le concept anthropologique de liminarité où les repères sont abolis. Masquant l'itération de ses voyages, Butler efface les traces des phases répétées de séparation et d'agrégation pour ne garder qu'un condensé de liminarité. La phase de liminarité y est présentée non plus comme une expérience à la marge, mais comme un tout autonome. C'est ce phénomène que distingue Van Guennep: «En outre, dans certains cas le schéma se dédouble: cela lorsque la marge est assez développée pour constituer une étape autonome.» (1981: 14). Butler parvient ainsi à faire de ce lieu de passage, de liminarité, un lieu d'ancrage dont il peut dès lors librement écrire la géographie.

\section{Cartographie et frontières : redéfinition}

\section{Du projet de l'écrivain à l'errance du voyageur}

Rappelons que Alps and Sanctuaries of Piedmont and the Canton Ticino s'ouvre sur une suite de contradictions entre l'objet annoncé de l'ouvrage et son contenu. Pour commencer, Butler présente des excuses au lecteur car malgré le titre, il ne consacrera pas une page à l'édifice religieux principal du Piémont, le Sacro Monte à Varallo :

I should perhaps apologise for publishing a work which professes to deal with the sanctuaries of Piedmont, and saying so little about the most important of them all-the Sacro Monte of Varallo. My excuse must be, that I found it impossible to deal with Varallo without making my book too long. Varallo requires a work to itself; I must, therefore, hope to return to it on another occasion. $(\mathrm{v})^{5}$

Plus loin, dans l'introduction, Butler fait l'éloge de l'Italie comme sa seconde patrie et annonce qu'il va y consacrer l'ouvrage complet : « during the remainder of my book I shall be occupied almost exclusively with Italy [...]. I have chosen Italy as my second country, and would dedicate this book to her as a thank-offering for the happiness she has afforded me.» (7) Or, sur les vingt-cinq communes qu'il visite, treize sont situées en Suisse dans le canton italophone du Tessin, deux sont en Lombardie, et dix sont en effet des communes piémontaises. Butler donne un titre précis à son œuvre, mais le discours qu'il tient dans la 
préface, puis dans l'introduction ne concorde pas avec le reste de l'ouvrage. Il redéfinit donc les frontières à sa convenance car s'il souhaite passer ses vacances en Italie, le voyage est une épreuve qu'il préfère écourter : "I was attracted to this place, in the first instance, chiefly because it is one of the easiest places on the Italian side of the Alps to reach from England. » (22) L'étymologie commune de travel et travail soulignée par Jean Viviès, trouve ici à nouveau tout son sens. Le trajet semble si long à l'écrivain qu'il propose une autre Italie, plus proche, le canton italophone du Tessin en Suisse. Il choisit un découpage géographique personnel, en cartographiant la région qui l'intéresse sur la base d'une unité culturelle (à son sens) et linguistique, mieux adaptée à ce qu'il recherche. Butler amende la cartographie selon ses propres repères: «The Vaudois, or Waldenses, though on the Italian side of the Alps, are French in language and perhaps in origin. » (111) L'Italie est donc surtout une vision qu'il compose dont il accommode la géographie.

Sans se soucier des frontières politiques, Butler personnalise sa cartographie de l'Italie en la fondant sur les reliefs et les dépressions naturelles des Alpes avec le col du SaintGothard comme frontière, ou point de passage et, de l'autre côté, le Val Léventine et brièvement le Val de Suse comme zones d'ancrage. C'est en ce sens que Butler se rapproche de l'étude physique de la géographie des Alpes. Cette posture est pour le moins avant-gardiste car elle correspond à un retour aux réalités naturelles du paysage et des habitants de la région pour lesquels des institutions ont depuis été créées, comme le Mountain Institute fondé par Elizabeth A. Byers (1972) ou l'ANEM en France (Association Nationale des Élus de Montagne) fondée en 1984.

31 Cependant, Butler n'a pas de projet géopolitique pour le Saint-Gothard ni pour le Val Léventine. Il pose ses repères pour définir un territoire qui lui soit propre, non pas celui d'une communauté au sens où l'entendent les géographes: "La communauté est une notion heuristique, qui permet de décrypter les logiques d'affiliation collective à un temps $t$, dans un environnement donné. » (Gouëset, Hoffmann, 2006 : 4). De l'autre côté de la montagne, comme à son habitude, il explore un espace peu fréquenté afin de se l'approprier en proposant une géographie toute personnelle.

\section{Coexistence entre passage et ancrage}

32 Après le passage du col du Saint-Gothard, Butler poursuit sa phase de séparation en cherchant à éviter les lieux touristiques. S'il voyage en compagnie de son ami Jones, il ne souhaite pas visiter des endroits prisés par les touristes britanniques, ni même par les touristes locaux, tant qu'il peut l'éviter. Peter Raby note à ce propos: «Initially, Butler went on holiday by himself. [...] After Jones had joined him for that first holiday in 1878, the pattern was repeated year after year, though Butler still normally spent some period of his own. » $(1991$ : 193) Son discours traduit régulièrement cet évitement: "During the whole time I was in that neighbourhood I saw not a single English, French, or German tourist. The ways of the inn, therefore, were exclusively Italian, and I had a better opportunity of seeing the Italians as they are among themselves than I ever had before." (Butler, 162). Les anglophones qu'il rencontre sont plutôt des immigrés qui sont partis en Grande-Bretagne ou aux États-Unis et dont les enfants sont bilingues. Les villages qu'il choisit sont à contre-courant de sa démarche car ce sont des villages que l'on quitte, des lieux d'émigration. Ils n'attirent pas les touristes et sont surtout décrits comme des lieux de passage comme en témoigne la construction du chemin de fer pour rejoindre l'Italie. Butler s'ancre donc dans une zone de liminalité où l'on ne s'arrête que rarement. Elle devient pour lui à la fois un point d'ancrage et une 
zone d'errance. Ce double attribut est la condition même de son appréhension renouvelée de l'espace et de sa représentation.

La liberté d'exploration est la caractéristique principale du discours dans Alps and Sanctuaries. Si les titres des chapitres permettent de retracer globalement ses itinéraires, seuls les villes ou les villages les plus importants sont reportés sur les cartes dans les guides de voyages principaux comme le Baedaker's. À partir de ces points d'ancrage, Butler découvre des villages moins fréquentés qu'il a donc la primeur de représenter, surtout pour le lecteur de l'époque. Il donne d'abord une vue très globale des lieux, puis il passe brutalement à une très petite échelle: "The sanctuary of Graglia is reached in about two hours from Biella. There are daily diligences. It is not so celebrated as that of Oropa, nor does it stand so high above the level of the sea, but it is a remarkable place and well deserves a visit. " (189) Puis il ajoute: "The restaurant is perfect-the best, indeed, that I ever saw in North Italy, or, I think, anywhere else. I had occasion to go into the kitchen, and could not see how anything could beat it for the most absolute cleanliness and order. » (189) Le passage d'une échelle trop grande à une échelle trop petite permet rarement au lecteur de reconstruire une image des lieux. Le métatexte confirme sa méthode de connaissance de l'espace non pas seulement par l'expérience, mais surtout par la trace qu'il a voulu en garder: "I find nothing more in my notes about Giornico except that the people are very handsome, and, as I thought, of a Roman type. » (76) Seules restent des impressions, qui sont celles de Butler. Les impressions collectées sur son passage ou les détails sur lesquels il s'arrête plus longuement décrivent sa façon de concevoir une géographie qui n'a pas vocation à aider au repérage spatial. C'est l'imprégnation plus ou moins intense d'un lieu chez le sujet voyageur qui permet d'accéder à sa connaissance.

\section{Conclusion}

Petit fils de géographe, Samuel Butler ne prend rien pour acquis et ses questionnements se traduisent par la recherche d'un ailleurs et d'une altérité que la montagne incarne de manière édifiante. S'il est parvenu à explorer des passages encore non-cartographiés en Nouvelle-Zélande, il va au-delà de cette démarche dans les Alpes. Butler voyage à contrecourant et ses vacances s'inscrivent dans une vacance qui participe d'une volonté d'" anti-tourisme ", c'est-à-dire la recherche d'une expérience personnelle du voyage et de la découverte qui n'est pas encadrée et/ou préconstruite par des guides ou des agences de voyage. À l'appui d'une composition originale, Butler invite le lecteur à partager son expérience de la liminarité. Il l'invite à abandonner ses repères spatio-temporels et lui en propose de nouveaux. Ceux-ci font peu cas de la chronologie, des distances et des échelles. Le rythme y est scandé par la musique qu'il choisit et le dessin du détail se substitue à l'échelle unique et toujours bien spécifiée de la carte de géographie.

Cette libération de toute démarche scientifique et de tout canon discursif permet l'émergence d'une nouvelle cartographie. Sous sa plume, travel et travail cessent d'aller de pair et le plaisir vient s'imposer comme seul guide de l'appréhension de l'espace. Butler explore dans une démarche de l'errance des lieux que l'on quitte ou par lesquels l'on transite. Dans un ouvrage à la forme et au discours expérimentaux, il vit une liminarité autonome qui définit une synonymie entre passage et ancrage. Ainsi Butler propose-t-il de nouvelles modalités d'accès à la connaissance de l'espace. 


\section{BIBLIOGRAPHIE}

BAEDEKER Karl (1897), Handbook for Travellers: Switzerland and the Adjacent Portion of Italy, Savoy, and Tyrol, Leipzig : Karl Baedeker Publisher.

BUTLER Samuel (1986), Alps and Sanctuaries of Piedmont and the Canton Ticino [1881], Londres : Allan Sutton.

Butler Samuel (1922), Erewhon; Or, Over the Range [1872], Londres : Page.

BUTLER Samuel (1922), The Way of all Flesh [1903], Londres : Cape.

CLIFFORD David, « Samuel Butler (1835-1902)», en ligne sur Victorianweb : < www.victorianweb.org/science/butler.html> (17 septembre 2015).

DeBARBIEUX Bernard \& FouRny Marie-Christine (2004), L'effet géographique. Construction sociale, appréhension cognitive et configuration matérielle des objets géographiques, Grenoble : Éditions de la MSH.

DEBARBIEUX Bernard (2001), «Les montagnes : représentations et constructions culturelles », Y. Veyret (dir.), Les montagnes : discours et enjeux géographiques, Paris : SEDES.

DeBARBIEUX Bernard (2008), « Mountains, Between Corporal Experience and Pure Rationality: Buache and Von Humboldt's Contradictory Theories », D. E. Cosgrove \& V. Della Dora (dir.), High Places: Cultural Geographies of Mountains, Ice and Science, Londres : Tauris.

DICKENS Charles \& ColLINS Wilkie (1990), « No Thoroughfare », No Thoroughfare and other Stories [1867], Phoenix Mill : Allan Sutton Publishing Limited, 1-127.

DICKENS Charles (2011), Little Dorrit [1855-7], Londres : Penguin.

FoURNY Marie-Christine (2008), « La frontière comme espace liminal. Proposition pour analyser l'émergence d'une figure de la frontière mobile dans le contexte alpin ", Journal of Alpine Research/ Revue de géographie alpine, <rga.revues.org/2115>.

GouËSET Vincent \& HoffMANN Odile (2006), « Communauté. Un concept qui semble poser problème dans la géographie française », R. Séchet \& V. Veschambre (dir.), Penser et faire la géographie sociale: Contribution à une épistémologie de la géographie sociale, <books.openedition.org/ pur/1918>.

Labex ITEM, projet TeLiMéP, en ligne sur <labexitem.fr/projet/telimep-territorialite-liminaliteet-metropolisation-peripheriques>.

MURRAY John (1852), A Handbook for Travellers in Northern Italy Embracing the Continental States, en ligne sur <archive.org/details/handbookfortrav18firgoog>.

Ounoughi Samia (2013a), « The Swiss Alps and Character framing in No Thoroughfare by Wilkie Collins and Charles Dickens, 1894 », M. LEROY (dir.), Dickens and Europe, New Castle upon Tyne : Cambridge Scholars Publishing, 114-125.

OUNOUGHI Samia (2013b) « Analyse discursive de la représentation des Alpes dans quelques œuvres de Charles Dickens ", conférence donnée lors du séminaire Geographies of displacement, Université Montpellier 3, Montpellier. 
OUNOUGHI Samia (2016), « La traversée des cols alpins : analyse du discours de la liminalité », E-rea (14.1 | 2016), <erea.revues.org/5531> (consulté le 02 janvier 2017).

PASCALI Adrien (1984), Le tour des horizons, Paris : Klincksieck.

RABY Peter (1991), Samuel Butler: a Biography, University of Iowa Press.

RADCLIFFE Ann (1992), The Romance of the Forest [1791], Oxford : Oxford University Press.

Roche Daniel (2003), Humeurs Vagabondes, de la circulation des hommes et de l'utilité des voyages, Paris : Fayard.

RUSKIN John (1982), Letters from the Continent [1858], Toronto : University of Toronto Press.

SWIFT Jonathan (1986), Gulliver's Travels [1721], Oxford : Oxford University Press.

Van GUENNEP Arnold (1981), Les Rites de Passage, Étude systématique des rites [1909], Paris : A. et J. Picard.

VIVIÈs Jean [dir.] (2002), Lignes d'Horizon, récits de voyage de la littérature anglaise, Aix-en-Provence : Publications de l'Université de Provence.

Viviès Jean (2015, février), « Le récit de voyage comme objet de recherche : questions génériques et critiques », conférence inaugurale du séminaire Passages, ancrage, Université Grenoble-Alpes, Grenoble.

VIVIÈs Jean (2016), Revenir/devenir. Gulliver ou l'autre voyage, Paris : Éditions de la rue d'Ulm.

ZDANSKI Clarice (2007), « Samuel Butler, Local Identity, and the Periodizing of Northern Italian Art: The Travel Writer-Painter's View of Art History ", J. Paradis (dir.), Samuel Butler, Victorian against the Grain: a Critical Overview, Toronto : University of Toronto Press.

\section{ANNEXES}

Samia Ounoughi est maître de conférences à l'Université Grenoble-Alpes et membre du LIDILEM. Ses travaux de recherche portent sur l'analyse du discours de la géographie, en particulier celle des montagnes. Son corpus inclut les textes de littérature, de voyage et des productions de géographes du xix ${ }^{e}$ siècle. Elle a publié plusieurs articles d'analyse du discours sur les Alpes de Samuel Butler et John Ruskin. Elle a édité La saillance et le discours sur le relief/Salience en Relief Related Discourse dans la Revue de Géographie alpine/Journal of Alpine Research (2016).

\section{NOTES}

1. L'ouvrage avec ses illustrations et ses partitions musicales est accessible en ligne : < archive.org/stream/alpssanctuarieso1882butl\#page/n13/mode/2up>

2. Page internet du projet TeLiMép dans le LABEX ITEM <labexitem.fr/projet/telimepterritorialite-liminalite-et-metropolisation-peripheriques>

3. Le deuxième voyage de Gulliver le mène chez un peuple de géants qui vit de l'autre côté d'une montagne infranchissable. Ce peuple se distingue par des règles de vie très différentes de celles que connaît Gulliver.

4. Le titre de l'œuvre No Thoroughfare de Dickens et Collins (1867) fait référence au col du Simplon. 


\section{RÉSUMÉS}

À la croisée de l'analyse du discours par les outils de la pragmatique, de la géographie constructiviste et de l'anthropologie, cet article explore comment les notions de passage et d'ancrage deviennent synonymes sous la plume de Samuel Butler dans son récit de voyages Alps and Santuaries of Piemont and the Canton Ticino (1881). Il montre comment le concept anthropologique de liminalité ou état d'entre-deux fait résonner la poétique de Butler et sert la géographie constructiviste actuelle. Il explore comment Butler déconstruit les canons de la littérature du tourisme et de la géographie physique pour nous offrir de nouvelles modalités d'accès à la connaissance des montagnes et de l'espace.

This cross-disciplinary article involves discourse analysis (pragmatics), subject-orientated geography and anthropology to reveal how the notions of crossing and anchoring become synonymous under Samuel Butler's quill in his travel book entitled Alps and Santuaries of Piemont and the Canton Ticino (1881). It shows how the anthropological concept of liminality -meaning the state of being in between states, echoes Butler's poetics while serving today's subject-orientated geography in offering us new ways and means to access knowledge of the mountains and more broadly of space.

INDEX

Mots-clés : Ancrage, cartographie, col, géographie, liminalité, montagne, passage, pragmatique, stylistique, voyage

Keywords : Anchoring, crossing, geography, liminality, mapping, mountains, pass, pragmatics, stylistics, travel

\section{AUTEUR}

\section{SAMIA OUNOUGHI}

LIDILEM (E.A. 609) 